\title{
Decursinol from Angelica gigas Nakai enhances endometrial receptivity during implantation
}

Seong-Eun Kim ${ }^{1 \dagger}$, Joo Eun Lee ${ }^{1 \dagger}$, Young-Hyun Han ${ }^{1 \dagger}$, Se-In Lee ${ }^{1}$, Do Kyung Kim², Seok-Rae Park ${ }^{1,3}$, Seong-Lan $\mathrm{Yu}^{1 *}$ and Jaeku Kang ${ }^{1,4^{*}}$ (D)

\begin{abstract}
Background: Embryo implantation is essential for a successful pregnancy, and an elaborate synchronization between the receptive endometrium and trophoblast is required to achieve this implantation. To increase 'endometrial receptivity', the endometrium undergoes transformation processes including responses of adhesion molecules and cellular and molecular cell to cell communication. Many natural substances from traditional herbs have been studied to aid in the achievement of successful implantation. In this study, we investigated positive effects on embryonic implantation with decursinol that is a major compound extracted from Angelica gigas Nakai known to be associated with promotion of healthy pregnancy in the traditional Korean herbal medicine.

Methods: Expression of cell adhesion molecules after treatment of endometrial epithelial cells by decursinol (40 or $80 \mu \mathrm{M}$ ) was determined using quantitative reverse transcription-polymerase chain reaction (qRT-PCR) and western blot analysis. The alteration of endometrial receptivity by decursinol ( 40 or $80 \mu \mathrm{M})$ was identified with the in vitro implantation model between Ishikawa cells and JAr cell spheroids (diameter, $143 \pm 16 \mu \mathrm{m}$ ). Exosomes secreted from Ishikawa cells after treatment of $80 \mu \mathrm{M}$ decursinol or dimethyl sulfoxide (DMSO) as the vehicle were investigated with invasion of JAr cells and attachment of JAr spheroids to Ishikawa cells.

Results: Decursinol significantly $(P<0.05)$ increased the expression of important endometrial adhesion molecules such as integrin $\beta 1, \beta 3, \beta 5$ and L-selectin mRNAs and integrin $\beta 5$ and L-selectin in protein. The adhesion rate of JAr spheroids to decursinol-treated Ishikawa cells also increased significantly which was 2.4-fold higher than that of the control $(P<0.05)$. Furthermore, decursinol induced an increase in the release of exosomes from Ishikawa cells and decursinol-induced exosomes showed autocrine (to Ishikawa cells) and paracrine (to JAr cells) positive effects on our implantation model.
\end{abstract}

Conclusion: These results propose that decursinol could serve as a new and alternative solution for patients who are infertile.

Keywords: Decursinol, Adhesion molecule, Integrin, Implantation, Endometrial receptivity, Exosomes

\footnotetext{
* Correspondence: yusl73@konyang.ac.kr; jaeku@konyang.ac.kr

†'Seong-Eun Kim, Joo Eun Lee and Young-Hyun Han contributed equally to this work.

${ }^{1}$ Priority Research Center, Myunggok Medical Research Institute, College of

Medicine, Konyang University, Daejeon 35365, Republic of Korea

Full list of author information is available at the end of the article
}

(c) The Author(s). 2020 Open Access This article is distributed under the terms of the Creative Commons Attribution 4.0 International License (http://creativecommons.org/licenses/by/4.0/), which permits unrestricted use, distribution, and reproduction in any medium, provided you give appropriate credit to the original author(s) and the source, provide a link to the Creative Commons license, and indicate if changes were made. The Creative Commons Public Domain Dedication waiver (http://creativecommons.org/publicdomain/zero/1.0/) applies to the data made available in this article, unless otherwise stated. 


\section{Background}

Implantation, a process whereby blastocysts attach to and invade the uterus endometrium, is an important gateway for a successful pregnancy. For a successful implantation, elaborate interaction between the developed trophoblast and the receptive endometrium is required [1]. The period termed 'window of implantation', which is the phase around day 9 after ovulation, only occurs when the uterus is ready to receive the conceptus $[2,3]$. Under regulation by estrogen (E2) and progesterone (P4), the endometrium undergoes numerous transformation processes, including responses of adhesion molecules, cellular and molecular cell-cell communication, extracellular matrix (ECM) remodeling, and expression of many growth factors, cytokines, and their mediators [4]. Integrin, one of the adhesion molecules, is considered to be important as a factor that regulates the establishment of uterine receptivity in general [5].

Failure to establish endometrial receptivity is a major cause of recurrent implantation failure in women $[6,7]$. To resolve this problem, assisted reproductive technologies (ART) have been steadily developed $[8,9]$. Many previous studies have sought substances such as calcitonin [10], low-dose aspirin [11], GnRH analogues [12] and natural substances extracted from Paeonia lactiflora [13], Perilla frutescens [14], Cyperus rotundus L. [15], Scutellaria baicalensis [16] that can help to increase endometrial receptivity. Since traditional herbal remedies have been proven to be safe when administered orally, many researchers have focused on natural substances [13-17]; however, failure of implantation still remains the main challenge in the success of ART $[8,9]$.

For thousands of years, the root of Angelica gigas Nakai (also known as Cham Dang Gui in Korea) has been used as a traditional Korean herbal medicine [18]. A. gigas is known to elicit various pharmacological effects including antiamnestic [19], platelet anti-aggregatory [20], anti-cancer [21-23], anti-inflammation [24], and antibacterial [18] activity. Additionally, it has already been proven to be highly safe with no chronic, hereditary, reproductive, or developmental toxicity when administered orally [25-27]. A. gigas is often referred to as the "female ginseng" [28] because of its extensive use to treat gynecological diseases such as dysmenorrhea, amenorrhea, menopausal syndromes, anemia, abdominal pain, injuries, migraine headaches, and arthritis [29-31]. Although A. gigas has traditionally been widely used for healthy pregnancy and easy delivery in China, Japan and Korea [31], due to the lack of suitable characterization, it remains unknown whether it has a positive effect on embryonic implantation. The major active compounds of $A$. gigas are essential oils ( $\alpha$-pinene, limonene, $\beta$-eudesmol, and elemol) and coumarins (decursinol, decursin, decursinol angelate, demethylsuberosin and nodakenindecursin) [32]. Coumarins extracted from plants have estrogenic activity since they show estrogen receptor relative binding affinities [33-35]. We performed a prescreening test (trophoblastic JAr cell adhesion) for coumarins derived from $A$. gigas and found that compared to decursin and decursinol angelate, decursinol was the compound most likely to increase the adhesion of trophoblast to the endometrium as it exhibits estrogen response element (ERE) activity [36]. Therefore, in this study, we aimed to determine the effect of decursinol on endometrial receptivity by assessing its effect on the adhesion phase of implantation. We also explored the mechanism by which it enhances endometrial receptivity.

\section{Methods \\ Material}

Decursinol, the single compound extracted from Angelica gigas Nakai was purchased from NPBANK of National Development Institute of Korean Medicine (Gyungsan, Korea); its molecular structure is shown in Fig. 1a. By performing HPLC chromatography, its purity was determined to be over $98.9 \%$.

\section{Cell lines and cell culture}

The human endometrial Ishikawa cell line derived from a human adenocarcinoma was kindly provided by Dr. Jacques Simard (CHUL Research Center, Quebec, Canada). Human choriocarcinoma JAr cells were obtained from the Korea Cell Line Bank (Seoul, Korea). Ishikawa cells were cultured in Dulbecco's modified Eagle's medium (DMEM; GE Healthcare, UK) with 10\% heat-inactivated fetal bovine serum (FBS; Gibco, Canada) and $1 \%$ penicillin / streptomycin. JAr cells were cultured in RPMI-1640 (GE Healthcare, UK) with 10\% heatinactivated FBS and $1 \%$ penicillin/streptomycin. Both cell lines were maintained as monolayers at $37^{\circ} \mathrm{C}$ in an atmosphere containing $5 \% \mathrm{CO}_{2} /$ air.

\section{Cell viability assay}

The cytotoxicity of decursinol was determined using an EZ-Cytox cell viability assay kit (Daeil Lab Service, Korea). Ishikawa cells were seeded in quadruplicate on a 48 -well cell culture plate at $8.0 \times 10^{4}$ cells/well density in a final volume of $0.5 \mathrm{~mL} /$ well DMEM medium with $10 \%$ FBS and incubated in a humidified $5 \% \mathrm{CO}_{2}$ incubator at $37^{\circ} \mathrm{C}$ for $24 \mathrm{~h}$. Each cell was treated with the indicated concentration of decursinol (from $5 \mu \mathrm{M}$ to $80 \mu \mathrm{M}$ ) incubated for $24 \mathrm{~h}$, followed by the addition of $50 \mu \mathrm{L} /$ well of EZ-Cytox reagent to both treated and control wells (without decursinol). After a $1 \mathrm{~h}$ incubation, the absorbance at $450 \mathrm{~nm}$ was measured with a microplate reader (Molecular Devices, California, USA). Corresponding blanks for each concentration were then used to validate the absorbance. The percentage of absorbance of treated cells was calculated against untreated cells. 
A<smiles>CC1(C)Oc2cc3oc(=O)ccc3cc2C[C@H]1O</smiles>

B

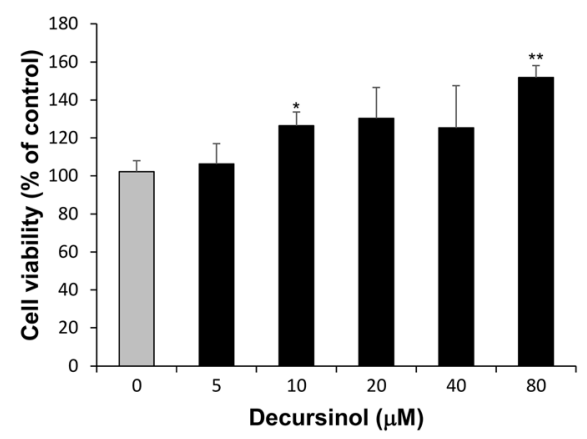

Fig. 1 Characterization of decursinol. a Molecular structure of decursinol. b Cytotoxic effect of decursinol on Ishikawa cells at the indicated concentrations for $24 \mathrm{~h}$. Values are expressed as mean \pm SD. The experiment was performed in quadruplicate well. ${ }^{*} P<0.05$ and ${ }^{* *} P<0.01$ are considered significant

Total RNA isolation and real-time qPCR

Ishikawa cells $\left(8.0 \times 10^{5}\right.$ cells/well $)$ were seeded into each 6-well cell culture plate in DMEM containing 10\% FBS. After a $24 \mathrm{~h}$ incubation, the culture fluid was replaced with serum-free medium and the cells treated with decursinol $(40$ or $80 \mu \mathrm{M})$ or DMSO as vehicle. After $24 \mathrm{~h}$, the culture fluid was removed and TRIzol reagent (Invitrogen, Thermo Fisher Scientific, Czech Republic) was added directly to each well. Total RNA was extracted according to the manufacturer's instruction and an equivalent amount of total RNA $(2 \mu \mathrm{g})$ from each sample reverse-transcribed using dNTP, oligo-dT primer and M-MLV reverse transcriptase (Promega, Madison, WI, USA). Real-time qPCR was performed on a real-time PCR detection system (BioRad, CA, USA) using IQ SYBR green chemistry (Bio-Rad). The amplification conditions for select genes (ITGB1, ITGB3, ITGB5, L-selectin, LIF, E-cadherin and $\beta$-actin) were: initial denaturation at $95^{\circ} \mathrm{C}$ for $30 \mathrm{~s}$, specific annealing temperature for $15 \mathrm{~s}$, and extension at $72^{\circ} \mathrm{C}$ for $15 \mathrm{~s}$ for 50 cycles. The homogeneity of the PCR amplification products was verified using the melting curve method. The sequences of the primers and the annealing temperature are given in Table 1. Expression levels of selected genes were quantified following normalization to the expression of $\beta$-actin. The results were calculated by the $2^{-\Delta \Delta \mathrm{CT}}$ method and fold change evaluated by comparison to the vehicle control. Each experiment was conducted at least in triplicate.

\section{Western blot analysis}

Ishikawa cells $\left(8.0 \times 10^{5}\right.$ cells/well $)$ were seeded into each 6well cell culture plate in DMEM containing 10\% FBS. After a $24 \mathrm{~h}$ incubation, the culture fluid was replaced with serum-free medium and cells were treated with decursinol $(40$ or $80 \mu \mathrm{M}$ ) or DMSO as vehicle. After $24 \mathrm{~h}$ of treatment, cells were washed with PBS and collected using Radioimmunoprecipitation (RIPA) solution. Protein quantification was performed by a BCA assay (Thermo Fisher

Table 1 PCR conditions, amplicon size for each target gene, and primers used in this study

\begin{tabular}{|c|c|c|c|}
\hline Gene & Primer sequences & Annealing temperature & Size (bp) \\
\hline ITGB1 & $\begin{array}{l}\text { Forward: 5'-GTCGTGTGTGTGAGTGCAAC-3' } \\
\text { Reverse: 5'-GCTGGGGTAATTGTCCCGA-3' }\end{array}$ & $60^{\circ} \mathrm{C}$ & 318 \\
\hline ITGB3 & $\begin{array}{l}\text { Forward: 5'-CTGCCGTGACGAGATTGAGT-3' } \\
\text { Reverse: 5'-TGCCCCGGTACGTGATATTG-3' }\end{array}$ & $60^{\circ} \mathrm{C}$ & 383 \\
\hline ITGB5 & $\begin{array}{l}\text { Forward: 5'-ACCTGGAACAACGGTGGAGA-3' } \\
\text { Reverse: 5'-AAAAGATGCCGTGTCCCCAA-3' }\end{array}$ & $60^{\circ} \mathrm{C}$ & 217 \\
\hline L-selectin & $\begin{array}{l}\text { Forward: 5'-ATTTCCTGGCACATCATG-3' } \\
\text { Reverse: 5'-ATTGTCTCGGCAGAATCT-3' }\end{array}$ & $56^{\circ} \mathrm{C}$ & 95 \\
\hline LIF & $\begin{array}{l}\text { Forward: 5'-ACAGAGCCTTTGCGTGAAAC-3' } \\
\text { Reverse: 5'-TGGTCCACACCAGCAGATAA-3' }\end{array}$ & $56^{\circ} \mathrm{C}$ & 78 \\
\hline E-cadherin & $\begin{array}{l}\text { Forward: 5'-GGCCTGAAGTGACTCGTAACG-3' } \\
\text { Reverse: 5'-TCAGACTAGCAGCTTCGGAACC-3' }\end{array}$ & $60^{\circ} \mathrm{C}$ & 201 \\
\hline$\beta$-actin & $\begin{array}{l}\text { Forward: 5'-CAAGAGATGGCCACGGCTGCT-3' } \\
\text { Reverse: 5'-TCCTTCTGCATCCTGTCGGCA-3' }\end{array}$ & 56 or $60^{\circ} \mathrm{C}$ & 275 \\
\hline
\end{tabular}


Scientific, Czech Republic). Equal amount of total protein ( $50 \mu \mathrm{g} / \mathrm{lane})$ was prepared and separated by sodium dodecyl sulfate-polyacrylamide gel electrophoresis (SDS-PAGE). The proteins were then transferred onto polyvinylidene difluoride (PVDF) membrane and blocked with 5\% skim milk in $1 \times$ TBST for $1 \mathrm{~h}$ at room temperature. Membranes were incubated with primary antibodies overnight at $4{ }^{\circ} \mathrm{C}$. Primary antibodies of integrin $\beta 1$ (cat. no. ab52971), $\beta 5$ (cat. no. ab184312), LIF (cat. no. ab113262), E-cadherin (cat. no. ab76055), CD9 (cat. no. ab2215), CD63 (cat. no. ab59479) were purchased from Abcam (Cambridge, UK); integrin $\beta 3$ (cat. no. PAB262Hu01) and L-selectin (cat. no. PAA086Hu01) from Cloud-Clone Corp. (Texas, USA); and GAPDH (cat. no. \#5174) from Cell Signaling Technology (MA, USA). After the reaction with appropriate secondary antibodies, all signals, except integrin $\beta 1$, were visualized using ECL chemiluminescence system (SuperSignal ${ }^{\mathrm{m}}$ West Pico PLUS Chemiluminescent Substrate, cat. no. 34577, Thermo Fisher Scientific, Czech Republic). Anti-integrin $\beta 1$ was visualized using ECL Select reagent (cat. no. \#RPN2235, GE Healthcare, UK). Western blot images were quantified by ImageJ software (http://imagej.nih.gov/ij/). Each experiment was performed at least in triplicate.

\section{Exosome isolation}

Ishikawa cells $\left(4.0 \times 10^{5}\right.$ cells $)$ were seeded into each 100-mm dish, in DMEM containing 10\% FBS. After a $24 \mathrm{~h}$ incubation, the culture media were changed to DMEM containing 1\% Bovine serum albumin (BSA; Sigma-Aldrich, Germany) and cells were treated with $80 \mu \mathrm{M}$ decursinol or DMSO as the vehicle. Twenty-four hours later, each culture medium was collected and centrifuged at $3000 \mathrm{~g}$ for $15 \mathrm{~min}$ to remove cells and cell debris. Supernatants were transferred to a sterile vessel. To isolate exosomes, ExoQuick ${ }^{\mathrm{Tm}}$ exosome precipitation solution (SBI System Biosciences, Inc., Mountain View, CA, USA) was added to the supernatants and the mixtures were incubated at $4{ }^{\circ} \mathrm{C}$ overnight. The mixtures were centrifuged at $1500 \mathrm{~g}$ for $30 \mathrm{~min}$. The pellets obtained were re-centrifuged ( $1500 \mathrm{~g}$ for $5 \mathrm{~min}$ ) and suspended in $80 \mu \mathrm{L}$ sterilized PBS. These isolated exosomes were quantified using EXOCET exosome quantitation kit (SBI System Biosciences, Inc., Mountain View, CA, USA) according to the protocol. The particle concentrations were $1.00 \times 10^{8}$ particles $/ \mu \mathrm{L}$ in exosomes isolated from decursinol-treated Ishikawa cells (D-Exo) and $4.96 \times 10^{7}$ particles $/ \mu \mathrm{L}$ in vehicle control (V-Exo).

\section{Cell adhesion assay}

Ishikawa cells $\left(1.5 \times 10^{5}\right.$ cells/well $)$ were seeded into 24 well cell culture plates with DMEM containing 10\% FBS and cultured for $24 \mathrm{~h}$. Medium was then replaced and the cells were incubated in serum-free DMEM containing decursinol ( 40 or $80 \mu \mathrm{M})$ or DMSO for $24 \mathrm{~h}$. JAr spheroids were made using a 96-well cell-repellent surface microplate (Greiner bio-one, Austria) according to the manufacturer's manual. JAr single cell suspensions (200 cells/ $100 \mu \mathrm{L}$ RPMI-1640 medium containing 10\% FBS and penicillin/streptomycin) were obtained by trypsinization of monolayer-cultured JAr cells and delivered into each well of the 96 well microplate prior to incubation in a humidified $5 \% \mathrm{CO}_{2}$ incubator at $37^{\circ} \mathrm{C}$ for $24 \mathrm{~h}$. JAr spheroids (20 spheroids/well) were then delivered onto a monolayer of Ishikawa cells in JAr growth medium. After incubation for $1 \mathrm{~h}$ at $37^{\circ} \mathrm{C}$, each well was washed with Dulbecco's Phosphate-Buffered Saline (DPBS; Thermo Fisher Scientific, Czech Republic) once, followed by the addition of JAr growth medium.

D-Exo and V-Exo were added to Ishikawa cells in serum-free DMEM. The JAr spheroids attachment was then examined in the same manner.

The attached JAr spheroids were visualized using a stereomicroscope (SZ 61, Olympus Corp, USA) and counted. Spheroids attachment is expressed as a percentage of seeded spheroids.

\section{Scanning electron microscope}

Pellets containing extracellular vesicles isolated from Ishikawa cells (i.e., exosomes) were vortexed and resuspended in $1 \mathrm{~mL}$ of distilled water. The solution was further purified using dialysis tubing cellulose membrane (molecular weight cut-off $=14,000$ ) against $1 \mathrm{~L} \times 3$ distilled water for 24 h. A $20 \mu \mathrm{L}$ solution was then placed on the center of the scanning electron microscope (SEM) sample holder $(\phi=15$ $\mathrm{mm}$ ) and dried in a ventilation hood for $30 \mathrm{~min}$. A $20 \mu \mathrm{L}$ volume of ethanol was then directly placed on the dried sample to dehydrate the exosomes. After evaporating the ethanol at room temperature for $30 \mathrm{~min}$, the sample holder was placed in a vacuum chamber for $2 \mathrm{~min}$ to eliminate any outgassing from the exosomes and water. To generate a conductive surface, a 10-nm gold coating was applied by sputtering (ion sputter coater) prior to imaging by SEM (SNE-4500 M, South Korea). SEM was used at $10.0 \mathrm{kV}$ and the images obtained were assessed using the Image J software (http://imagej.nih.gov/ij/) software for analysis of exosome size.

\section{Invasion assay}

JAr cell invasion assay was performed by measuring the invasion of cells through Matrigel (BD Bioscience, San Jose, CA) in a 48-well micro chemotaxis chamber (Neuroprobe, Gaithersburg, USA). For the invasion assay, a single $25 \times 80 \mathrm{~mm}$ piece of Polycarbonate track-etch (PCTE) filter membrane with a $12-\mu \mathrm{m}$ pore size was coated with a final concentration of $10 \mu \mathrm{g} / \mathrm{mL}$ of Matrigel in serum-free RPMI 1640. Lower wells of the chamber were filled with $30 \mu \mathrm{L}$ of medium containing 10\% FBS which served as the chemoattractant. The chemotaxis chamber was assembled by placing the filter membrane between the lower and 
upper chamber with the Matrigel-coated side up. A suspension of $5.0 \times 10^{5}$ cells $/ \mathrm{mL}$ was treated with $2 \mu \mathrm{L}$ of isolated exosomes per mL with $50 \mu \mathrm{L}$ placed in each well of the upper chamber. After incubation for $11 \mathrm{~h}$ at $37^{\circ} \mathrm{C}$ in a $5 \% \mathrm{CO}_{2}$ incubator, filters were removed, fixed, and stained with Diff-Quik reagents (Sysmex Corporation, Kobe, Japan). Before staining and counting of migrated cells could be performed, non-migrated cells were removed from the top side of the filter. The number of JAr cells that invaded into the lower surface of the filters was counted in 3 random fields under a light microscope at $x$ 200 magnification.

\section{Statistical analysis}

For parametric data, comparisons between groups were performed using analysis of variance (ANOVA) and statistically significant differences were calculated by student t-test, using $\mathrm{R}$ program and GraphPad Prism (GraphPad Software, USA). The minimum significance level was a $P$ value of 0.05 . All experiments were independently performed at least in triplicate.

\section{Results}

\section{Cytotoxicity of decursinol}

A WST-1-based cell viability assay was used to examine the toxicity of decursinol on Ishikawa cell at various concentrations. Although decursinol did not exhibit any cytotoxic effect on Ishikawa cells (Fig. 1b), it displayed a slight proliferative effect at high concentrations. Therefore, we selected the concentrations, $40 \mu \mathrm{M}$ and $80 \mu \mathrm{M}$, for the experimental group.

\section{Effects of decursinol on the expression of endometrial receptivity markers}

Integrins, L-selectin, and E-cadherin are known as adhesion molecules expressed in human endometrial epithelial cells. LIF is also known as an endometrial receptivity marker that increases the expression of integrin $\beta 3$ and $\beta 5$ $[11,37]$. To assess the effect of decursinol on the expression of these receptivity markers in endometrial epithelial cells, Ishikawa cells were treated with different concentrations of decursinol $(40 \mu \mathrm{M}$ and $80 \mu \mathrm{M})$ and the mRNA expression of receptivity markers measured via real-time qPCR. Integrin $\beta 1$ and L-selectin were increased significantly when cells were treated with $80 \mu \mathrm{M}$ decursinol for $12 \mathrm{~h}$ (Fig. 2a, d). Similarly, integrin $\beta 3$ and integrin $\beta 5$ mRNA levels were significantly increased (greater than two folds) after treatment with 40 and $80 \mu \mathrm{M}$ decursinol at $24 \mathrm{~h}$ (Fig. 2b, c). Although integrin $\beta 3$ and integrin $\beta 5$ showed increased expression, LIF level was significantly decreased only at $24 \mathrm{~h}$ after treatment with $40 \mu \mathrm{M}$ decursinol (Fig. 2e). E-cadherin expression showed no significant change at all concentrations and times (Fig. 2f). Protein expression were determined by a western blot analysis after decursinol treatment for $24 \mathrm{~h}$ (Fig. 3). The expression of integrin $\beta 5$ was significantly increased with 40 and $80 \mu \mathrm{M}$ decursinol treatment (Fig. 3c), while L-selectin expression showed significant increase with $80 \mu \mathrm{M}$ decursinol treatment (Fig. 3d).

\section{Effects of decursinol on the attachment of JAr spheroid to Ishikawa cells}

Fig. 4a shows stereomicroscopic image of JAr spheroids formed with uniform shape. Diameters of JAr spheroids were similar to those of embryos at the period of implantation, with an average of $143 \pm 16 \mu \mathrm{m}$.

Decursinol significantly increased the attachment rate of JAr spheroids when treated at $80 \mu \mathrm{M}$ concentration (Fig. 4b). Adhesion rate was $35.0 \%$, a value 2.4 -fold higher than that for the control. There was no significant difference $(P>0.05)$ at the $40 \mu \mathrm{M}$ concentration.

\section{D-Exo enhances trophoblastic JAr cell invasion}

Exosomes were isolated from decursinol-treated Ishikawa cells to confirm their positive role in JAr-Ishikawa cell communication in implantation. Fig. 5a shows a SEM image of exosomes from Ishikawa cells. All exosomes had a spherical shape and an average size of $192.79 \pm 21.51 \mathrm{~nm}$. CD9 and CD63 are representative exosomal surface markers and western blot analysis confirmed the presence of exosomes in V-Exo and D-Exo (Fig. 5b). Based on prior quantification of the exosome particles, D-Exo appeared to contain more exosomes than V-Exo. In contrast, the marker proteins were not detected in FBS and culture medium (Fig. 5b). To determine whether D-Exo exhibits positive effects on JAr cells, an invasion assay was performed. The invasion rate of JAr cells increased significantly after treatment with D-Exo compared to V-Exo (Fig. 5c).

\section{D-Exo increases the rate of attachment of JAr spheroids to Ishikawa cells}

To determine the autocrine effect of D-Exo, a JAr spheroid adhesion assay was performed. The rate of attachment of JAr spheroids to V-Exo and D-Exo-treated Ishikawa cells was 38.3 and $59.3 \%$ respectively; these values were 1.5 -fold greater than that for the control (Fig. 5d). This result demonstrates that not only decursinol itself, but decursinol-induced exosomes also significantly increased the attachment rate of JAr spheroids.

\section{Discussion}

In this study, we determined the effect of decursinol on endometrial receptivity by assessing its effect on the adhesion phase of implantation. We also explored the mechanism by which it enhances endometrial receptivity. We found that decursinol increased the mRNA expression of integrin $\beta 1, \beta 3, \beta 5$ and L-selectin (Fig. 2), the 
A

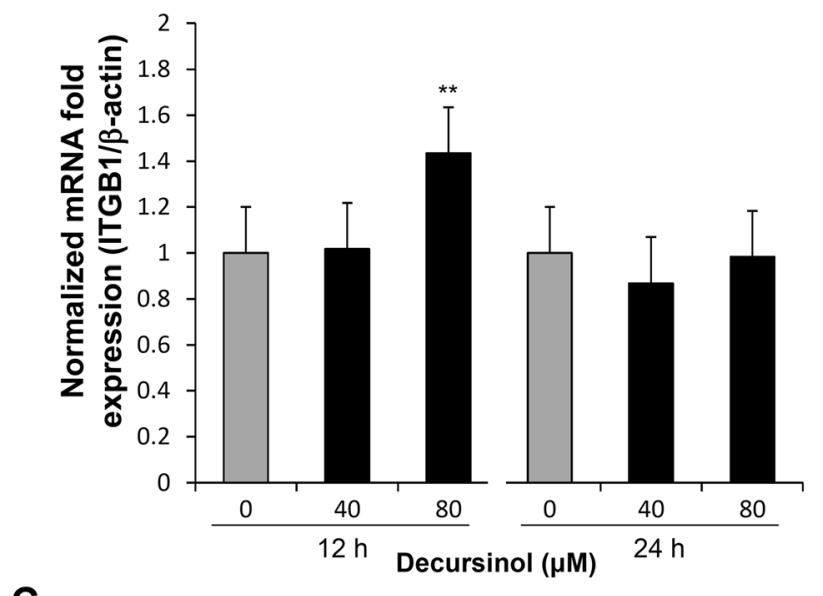

C

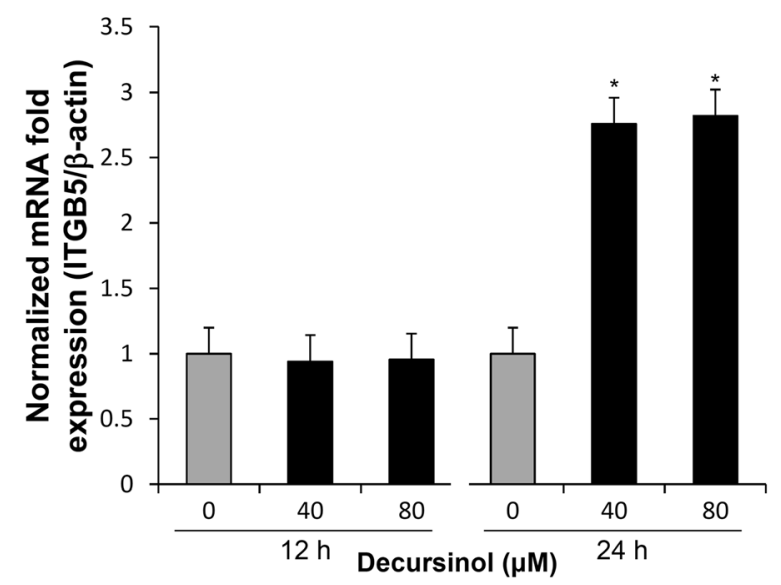

$E$

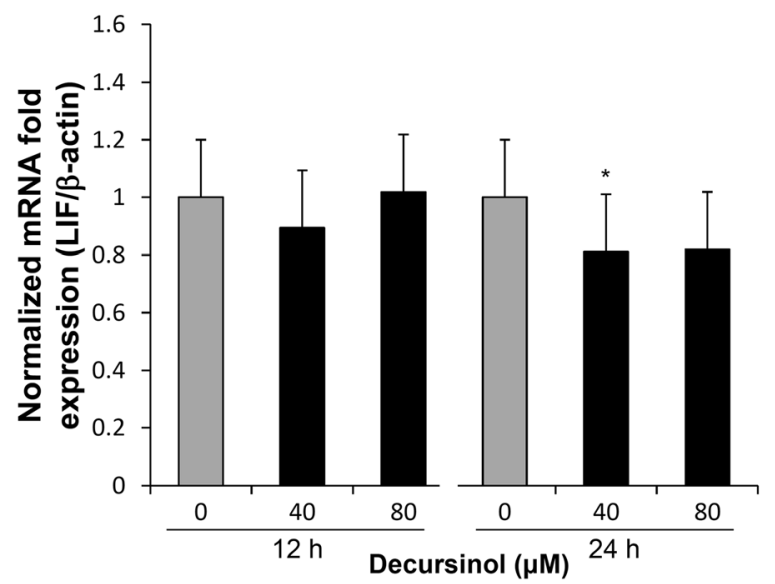

B
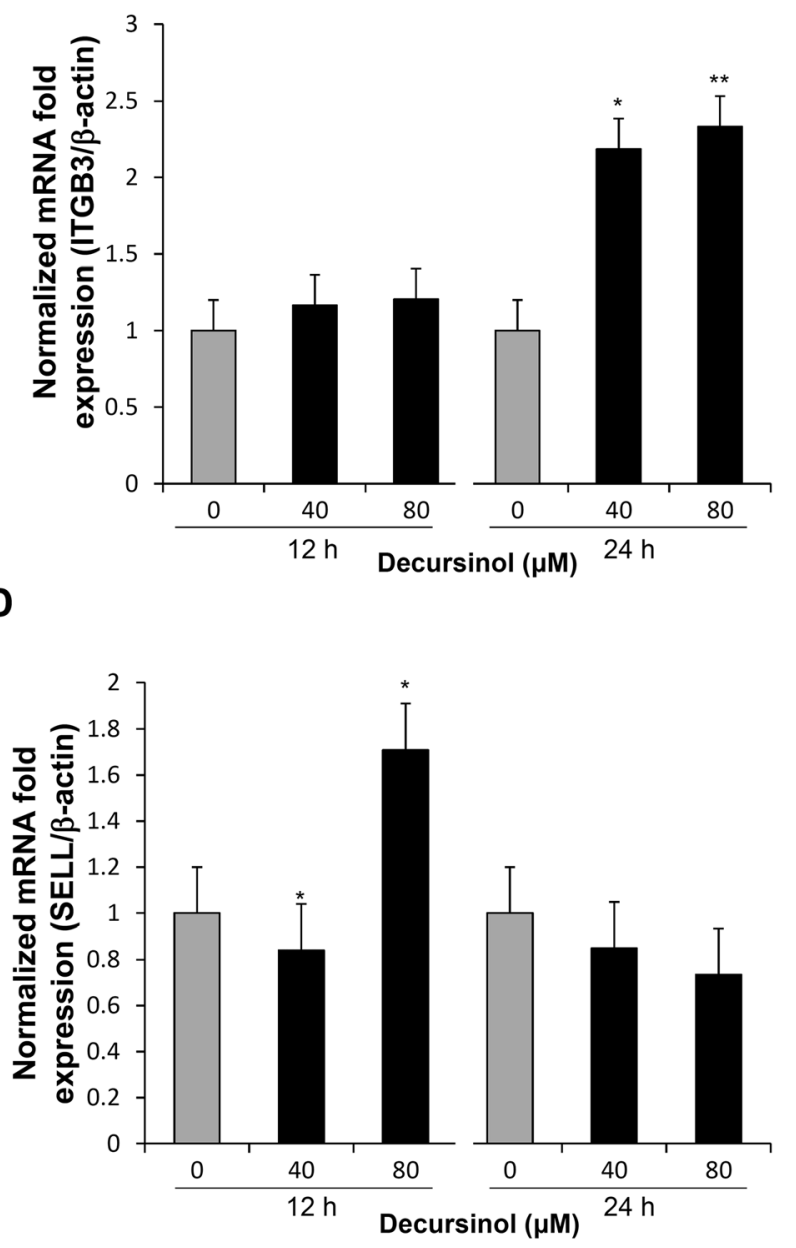

$\mathbf{F}$

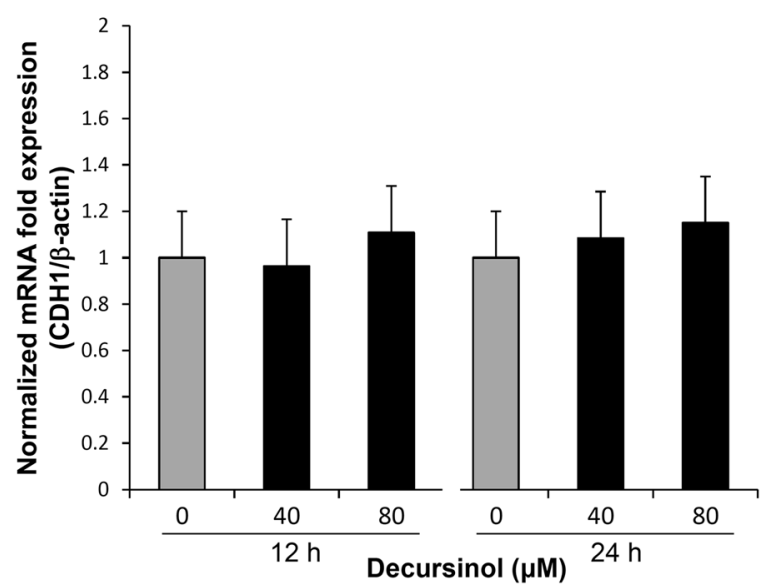

Fig. 2 Real-time qPCR was performed to assess the effect of decursinol on the mRNA expression of ITGB1, ITGB3, ITGB5, L-selectin, LIF, and Ecadherin. a ITGB1, b ITGB3, c ITGB5, d L-selectin, e LIF, and f E-cadherin mRNA in the Ishikawa cells transfected at $12 \mathrm{~h}$ and $24 \mathrm{~h}$ following decursinol treatment ( 40 or $80 \mu \mathrm{M}$ ). Data shown are normalized mRNA fold change. Values are expressed as mean \pm SD. The experiments were repeated in triplicate wells. ${ }^{*} P<0.05$ and ${ }^{* *} P<0.01$ are considered significant 


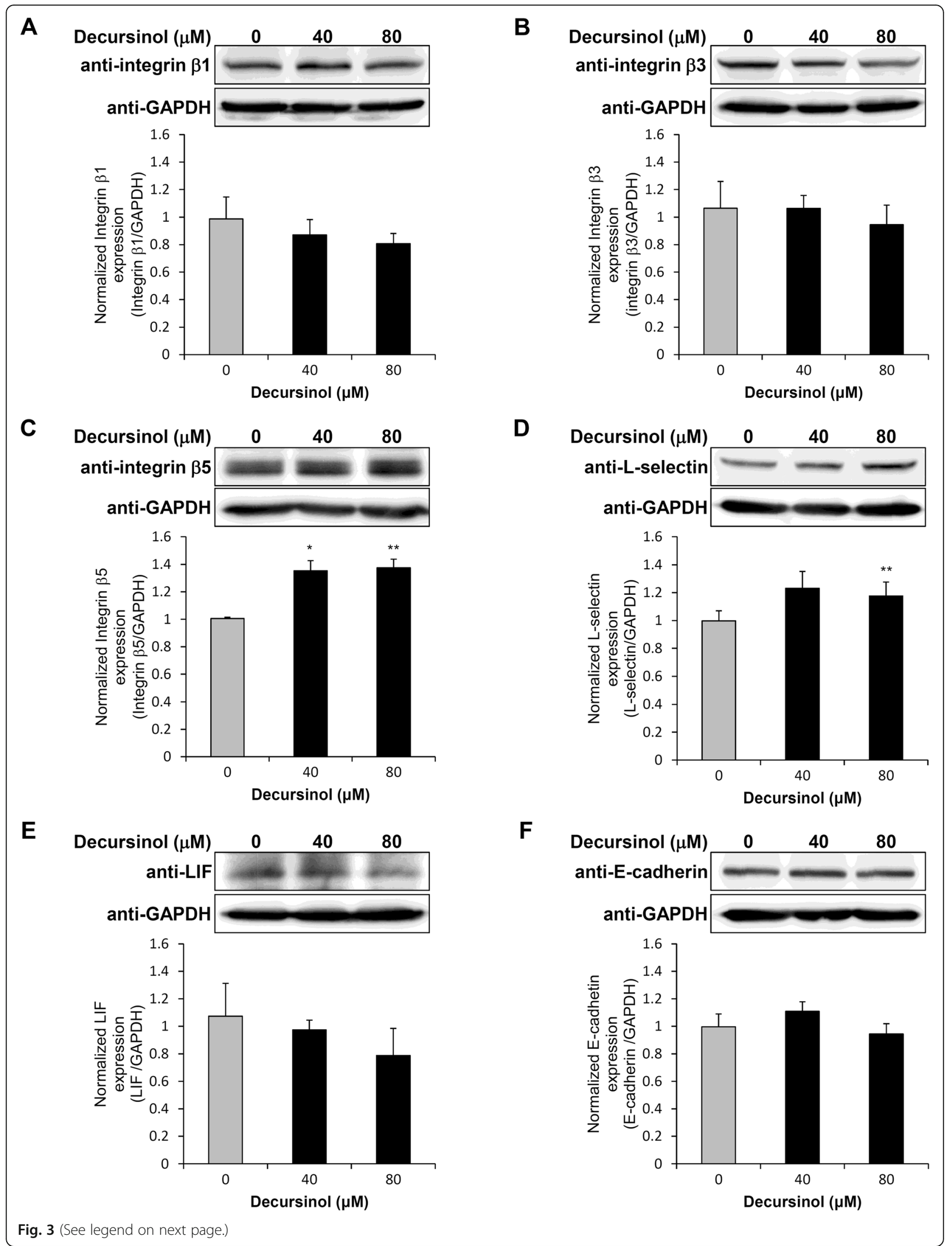


(See figure on previous page.)

Fig. 3 Western blot analysis was performed to assess the effect of decursinol on the protein expression of integrin $\beta 1$, integrin $\beta 3$, integrin $\beta 5$, Lselectin, LIF, and E-cadherin. a Integrin $\beta 1$, b integrin $\beta 3$, c integrin $\beta 5$, d L-selectin, e LIF, and $\mathbf{f} E$-cadherin expression on the Ishikawa cells were measured after $24 \mathrm{~h}$ of decursinol treatment $(40$ or $80 \mu \mathrm{M}$ ). Data are shown as normalized protein expression. Values are expressed as mean \pm SD. The experiments were repeated in quadruplicate wells. ${ }^{*} P<0.05$ and ${ }^{*} P<0.01$ are considered significant

protein expression of integrin $\beta 5$ and L-selectin (Fig. 3), and the endometrial adhesion of JAr spheroids (Fig. 4).

Implantation has three steps (apposition, adhesion, and invasion) with each involving complex signaling cascades that are essential for pregnancy. Molecular mediators of adhesion appear to have different action mechanisms or timing [38], and in our study, decursinol appeared to exhibit a different effect for each expression of the adhesion molecules (Figs. 2, 3). Although an increase in marker expression led to an increase in trophoblast attachment rate [39] (Fig. 4), further studies are required to determine the kinetic action mechanism of decursinol, which is the future direction of our research.

Although a study reported that the expression of integrin $\beta 3$ and $\beta 5$ was increases in an LIF-dependent manner [39], decursinol did not reveal an apparent LIF-dependent mechanism underlying the increase in integrin $\beta 3$ and $\beta 5$ expression in our study; relative to integrin expression, there was no increase in LIF expression (Figs. 2, 3). Our results also showed that the estrogen receptor inhibitor, ICI 182780, did not have an inhibitory effect on decursinol (data not shown), implying that decursinol might not be related to estrogen response element (ERE). Studies are however needed to determine whether the effect of decursinol on implantation is related to glucocorticoid receptors (GRs). Uterine GR signaling as a transcriptional regulator has been deemed important in regulating the immune system and inflammatory response [40] and pre-implantation anti-inflammatory therapies have been reported to increase pregnancy rates [41]. Since decursinol is well known for its anti-inflammatory effect as a coumarin extract [42], further investigations are required to determine whether its positive role in implantation is due to its antiinflammatory effects via GRs.

Glycosylation is an important post-translational modification of adhesion proteins involved in reproduction, especially in the implantation process [14, 43]. Certain changes in glycosylation of the uterine endometrial epithelial cells are necessary for successful embryonic adhesion to the endometrium [43, 44]. From our western blot results (Fig. 3), the observed band size of proteins such as integrin $\beta 1, \beta 3$, and L-selectin was found to be slightly higher than the predicted band size; therefore, decursinol is expected to have a glycosylation effect on adhesion proteins in the adhesion phase of implantation. Further studies investigating the function of and glycosylation patterns induced by decursinol will be needed to provide meaningful insights into the actual biological mechanism.

Exosomes released from endometrial epithelial cells play an important role in endometrial-blastocyst interactions during embryo implantation [6]. Exosomes found in endometrial fluid and follicular fluid of ovarian follicles contain microRNAs and exosome- and cell-type-specific proteins. Therefore, these exosomes can mediate cell communication within the blastocyst and endometrium and regulate trophoblast maturation [45-47]. Accordingly, we were
A

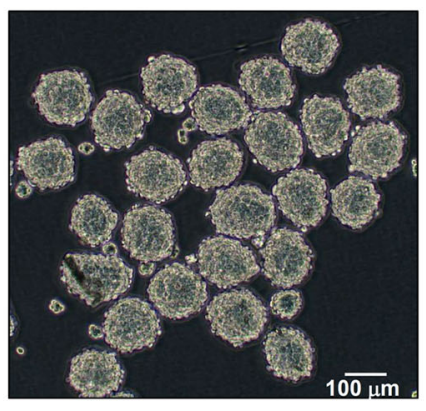

B

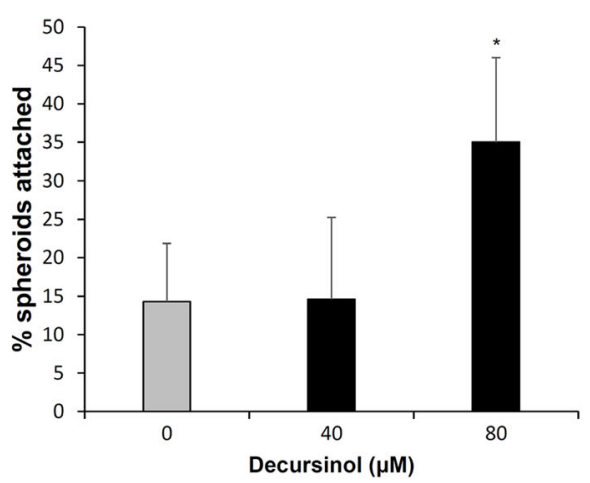

Fig. 4 Assessment of the decursinol concentration-dependent attachment rate of JAr spheroids to Ishikawa monolayers. a Representative image of cultured JAr spheroids taken using a stereo-microscope at magnification $\times 100$. $\mathbf{b}$ Attached JAr spheroids were manually counted and the attachment rate was expressed as mean \pm SD. The experiment was repeated in quadruplicate wells. ${ }^{*} P<0.05$ is considered significant 


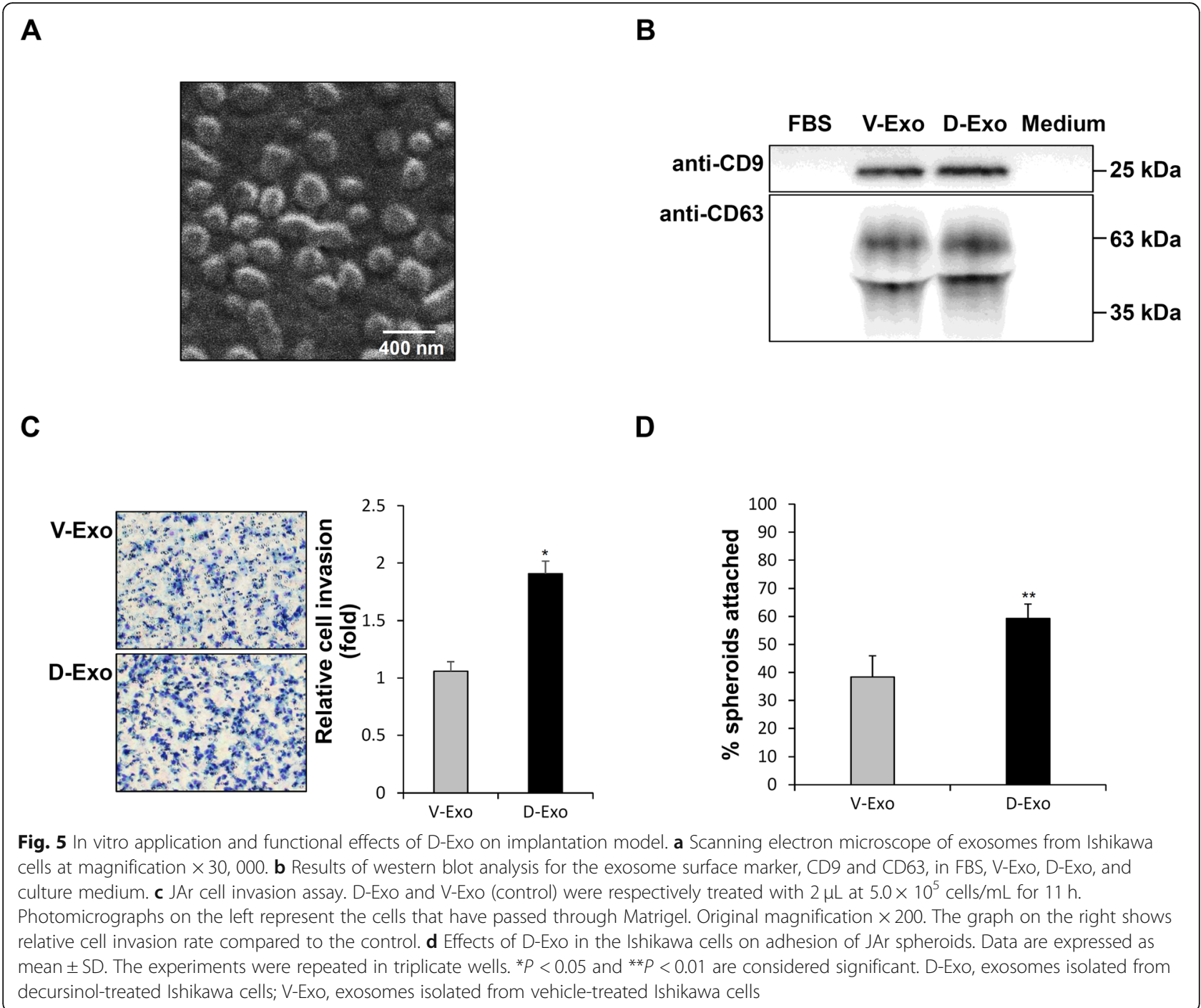

interested in the effect of exosomes in the adhesion phase of implantation and thus, sought to explore this molecular aspect. Decursinol-induced exosomes displayed positive effects on both recipient cells (JAr cells) and parent cells (Ishikawa cells) (Fig. 5c, d). Prior studies reported that exosomes can only exhibit positive effects on recipient cells, and not on parent cells (same cell type that the exosome was isolated from) [48]. However, our study paves the way for investigation on a possible autocrine effect being exhibited by decursinol. This is because the exosomes obtained from decursinol-treated Ishikawa cells can affect the same cells in their surroundings. This finding is meaningful since decursinol can induce a positive feedback, leading to a stepwise effect of progressively increasing endometrial receptivity. Decursinol-treated Ishikawa cells released $1.00 \times 10^{8}$ particles $/ \mu \mathrm{L}$, which is about $100 \%$ increase compared to the control $\left(4.96 \times 10^{7}\right.$ particles $\left./ \mu \mathrm{L}\right)$. As shown in Fig. $1 \mathrm{~b}$, decursinol has a proliferative effect on Ishikawa cells, increasing the number of Ishikawa cells up to about $50 \%$. Therefore, we assume that among 100\% increase of exosome amount, about $50 \%$ was due to increase in number of Ishikawa cells, and the other about $50 \%$ was solely due to direct effect of decursinol on secreting ability of Ishikawa cells. Which means that direct effect of decursinol on exosome secretion resulted in $2.50 \times 10^{7}$ particles $/ \mu \mathrm{L}$ increase of exosomes. Assessing whether decursinol also has an effect on changing the content of exosomes will be our next focus. In addition, in order to elucidate the precise action mechanism of exosomes, microarray analysis should be conducted to derive a profile for the contents of the exosomes. Further studies should be conducted by labeling specific microRNAs and proteins based on their abundance in exosomes to determine their specific functions.

To determine whether implantation rate changes in response to decursinol, an additional in vivo experiment should be performed using an implantation failure 
mouse model $[14,49]$. An in vivo model should also be utilized, with methods similar to those used in our study, for analysis of changes in the expression of endometrial receptivity markers; this would contribute to the possibility of decursinol being employed as a new and alternative therapy for patients who are infertile.

\section{Conclusion}

To summarize, decursinol increased not only the expression of the endometrial adhesion molecules (integrin $\beta 5$ and L-selectin), but also the rate of adhesion between JAr spheroids and the Ishikawa monolayer and exosome secretion from Ishikawa cells. In addition, these decursinolinduced exosomes had both autocrine (to Ishikawa cells) and paracrine (to JAr cells) positive effects. These results suggest that decursinol could function as a new and alternative therapy by increasing the success rate of implantation in the adhesion phase.

\section{Abbreviations}

ART: Assisted reproductive technologies; ECM: Extracellular matrix;

ERE: Estrogen response element; GRs: Glucocorticoid receptors

\section{Acknowledgments}

None.

\section{Authors' contributions}

SEK, JEL and YHH: data collection, data management, data analysis and manuscript writing. SIL and DKK: data collection. SRP: project development and manuscript editing. SLY: project development, data collection, data management and manuscript editing. JKK: project development, data management and manuscript editing. All authors read and approved the final manuscript.

\section{Funding}

This work was supported by the Basic Science Research Program through the National Research Foundation, the Ministry of Education, Korea (NRF2017R1A6A1A03015713). This research was performed under the Student Research Internship Program through College of Medicine, Konyang University. Both funding bodies provide financial support and did not involve in the design of the study and the collection, analysis, interpretation of data and in writing the manuscript.

\section{Availability of data and materials}

The raw data for this study is available upon reasonable request to the corresponding author.

\section{Ethics approval and consent to participate}

This article does not contain any studies with human participants or animals performed by any of the authors.

\section{Consent for publication}

Not applicable.

\section{Competing interests}

The authors declare that they have no conflict of interest.

\section{Author details}

${ }^{1}$ Priority Research Center, Myunggok Medical Research Institute, College of Medicine, Konyang University, Daejeon 35365, Republic of Korea.

${ }^{2}$ Department of Anatomy, College of Medicine, Konyang University, Daejeon 35365, Republic of Korea. ${ }^{3}$ Department of Microbiology, College of Medicine, Konyang University, Daejeon 35365, Republic of Korea. ${ }^{4}$ Department of Pharmacology, College of Medicine, Konyang University, Daejeon 35365, Republic of Korea.
Received: 30 April 2019 Accepted: 20 January 2020

Published online: 05 February 2020

\section{References}

1. Koot YE, Macklon NS. Embryo implantation: biology, evaluation, and enhancement. Curr Opin Obstet Gynecol. 2013;25(4):274-9.

2. Dey SK, Lim H, Das SK, Reese J, Paria BC, Daikoku T, Wang H. Molecular cues to implantation. Endocr Rev. 2004;25(3):341-73.

3. Wang H, Dey SK. Roadmap to embryo implantation: clues from mouse models. Nat Rev Genet. 2006;7(3):185-99.

4. Greening DW, Nguyen HP, Elgass K, Simpson RJ, Salamonsen LA. Human endometrial Exosomes contain hormone-specific cargo modulating Trophoblast adhesive capacity: insights into endometrial-embryo interactions. Biol Reprod. 2016;94(2):38.

5. Lessey BA. Endometrial integrins and the establishment of uterine receptivity. Hum Reprod. 1998;13(Suppl 3):247-58 discussion 259-261.

6. Teh WT, McBain J, Rogers P. What is the contribution of embryo-endometrial asynchrony to implantation failure? J Assist Reprod Genet. 2016;33(11):1419-30.

7. Valdes CT, Schutt A, Simon C. Implantation failure of endometrial origin: it is not pathology, but our failure to synchronize the developing embryo with a receptive endometrium. Fertil Steril. 2017;108(1):15-8.

8. Polanski LT, Baumgarten MN, Quenby S, Brosens J, Campbell BK, RaineFenning NJ. What exactly do we mean by 'recurrent implantation failure'? A systematic review and opinion. Reprod BioMed Online. 2014;28(4):409-23.

9. European IVFmC, European Society of Human R, embryology, Calhaz-Jorge C, De Geyter C, Kupka MS, de Mouzon J, Erb K, Mocanu E, Motrenko T et al: assisted reproductive technology in Europe, 2013: results generated from European registers by ESHRE. Hum Reprod 2017, 32(10):1957-1973.

10. Xiong T, Zhao Y, Hu D, Meng J, Wang R, Yang X, Ai J, Qian K, Zhang H. Administration of calcitonin promotes blastocyst implantation in mice by up-regulating integrin beta3 expression in endometrial epithelial cells. Hum Reprod. 2012;27(12):3540-51.

11. Zhao M, Chang C, Liu Z, Chen LM, Chen Q. Treatment with low-dose aspirin increased the level LIF and integrin beta3 expression in mice during the implantation window. Placenta. 2010;31(12):1101-5.

12. Li F, Zhang M, Zhang Y, Liu T, Qu X. GnRH analogues may increase endometrial Hoxa10 promoter methylation and affect endometrial receptivity. Mol Med Rep. 2015;11(1):509-14.

13. Choi HJ, Chung TW, Park MJ, Lee KS, Yoon Y, Kim HS, Lee JH, Kwon SM, Lee SO, Kim KJ, et al. Paeonia lactiflora enhances the adhesion of Trophoblast to the endometrium via induction of leukemia inhibitory factor expression. PLoS One. 2016;11(2):e0148232.

14. Kim EY, Choi HJ, Chung TW, Choi JY, Kim HS, Jung YS, Lee SO, Ha KT. Waterextracted Perilla frutescens increases endometrial receptivity though leukemia inhibitory factor-dependent expression of integrins. J Pharmacol Sci. 2016;131(4):259-66.

15. Choi HJ, Chung TW, Park MJ, Jung YS, Lee SO, Kim KJ, Ha KT. Waterextracted tubers of Cyperus rotundus L. enhance endometrial receptivity through leukemia inhibitory factor-mediated expression of integrin alphaVbeta3 and alphaVbeta5. J Ethnopharmacol. 2017;208:16-23.

16. Zhang YM, Zhang YY, Bulbul A, Shan X, Wang XQ, Yan Q. Baicalin promotes embryo adhesion and implantation by upregulating fucosyltransferase IV (FUT4) via Wnt/beta-catenin signaling pathway. FEBS Lett. 2015;589(11):1225-33.

17. Kim SJ, Ko SM, Choi EJ, Ham SH, Kwon YD, Lee YB, Cho HY. Simultaneous Determination of Decursin, Decursinol Angelate, Nodakenin, and Decursinol of Angelica gigas Nakai in Human Plasma by UHPLC-MS/MS: Application to Pharmacokinetic Study. Mol. 2018;23(5):E1019.

18. Lee S, Shin DS, Kim JS, Oh KB, Kang SS. Antibacterial coumarins from Angelica gigas roots. Arch Pharm Res. 2003;26(6):449-52.

19. Kang SY, Lee KY, Park MJ, Kim YC, Markelonis GJ, Oh TH, Kim YC. Decursin from Angelica gigas mitigates amnesia induced by scopolamine in mice. Neurobiol Learn Mem. 2003;79(1):11-8.

20. Lee YY, Lee S, Jin JL, Yun-Choi HS. Platelet anti-aggregatory effects of coumarins from the roots of Angelica genuflexa and A. gigas. Arch Pharm Res. 2003;26(9):723-6.

21. Ahn KS, Sim WS, Kim IH. Decursin: a cytotoxic agent and protein kinase C activator from the root of Angelica gigas. Planta Med. 1996;62(1):7-9.

22. Lee HJ, Lee HJ, Lee EO, Lee JH, Lee KS, Kim KH, Kim SH, Lu J. In vivo anticancer activity of Korean Angelica gigas and its major pyranocoumarin decursin. Am J Chin Med. 2009;37(1):127-42. 
23. Yim D, Singh RP, Agarwal C, Lee S, Chi H, Agarwal R. A novel anticancer agent, decursin, induces $\mathrm{G} 1$ arrest and apoptosis in human prostate carcinoma cells. Cancer Res. 2005;65(3):1035-44.

24. Kim JH, Jeong JH, Jeon ST, Kim H, Ock J, Suk K, Kim Sl, Song KS, Lee WH. Decursin inhibits induction of inflammatory mediators by blocking nuclear factor-kappaB activation in macrophages. Mol Pharmacol. 2006;69(6):1783-90

25. Yun JW, Che JH, Kwon E, Kim YS, Kim SH, You JR, Kim WH, Kim HH, Kang BC. Safety evaluation of Angelica gigas: Genotoxicity and 13-weeks oral subchronic toxicity in rats. Regul Toxicol Pharmacol: RTP. 2015;72(3):473-80.

26. Kim KM, Lee YJ, Hong YG, Kang JS. Oral acute and subacute toxicity studies of decursin and decursinol angelate of Angelica gigas Nakai. Mol Cell Toxicol. 2009;5(2):153-9.

27. Oh SR, Ok S, Jung TS, Jeon SO, Park JM, Jung JW, Ryu DS. Protective effect of decursin and decursinol angelate-rich Angelica gigas Nakai extract on dextran sulfate sodium-induced murine ulcerative colitis. Asian Pac J Trop Med. 2017;10(9):864-70.

28. Sarker SD, Nahar L. Natural medicine: the genus Angelica. Curr Med Chem. 2004;11(11):1479-500.

29. Kim GS, Park CG, Jeong TS, Cha SW, Baek NI, Song KS. ACAT (acyl-CoA: cholesterol acyltransferase) inhibitory effect and quantification of pyranocurmarin in different parts of Angelica gigas Nakai. J Appl Biol Chem. 2009:52:188-94.

30. Park KW, Choi SR, Hong HR, Kim JY, Shon MY, Seo KI. Biological activities of methanol extract of Angelica gigas Nakai. Korean J Food Preserv. 2007;14:655-61.

31. Bae IY, Lee JY, Kwak BY, Lee HG. Estrogenic effects of various extracts from Chamdanggui (Angelica gigas Nakai) and Sogdan (Phlomis umbrosa Turcz). Food Sci Biotechnol. 2011;20(4):1113-8.

32. Lee HW, Ryu HW, Kang MG, Park D, Lee H, Shin HM, Oh SR, Kim H. Potent inhibition of monoamine oxidase a by decursin from Angelica gigas Nakai and by wogonin from Scutellaria baicalensis Georgi. Int J Biol Macromol. 2017;97:598-605

33. Choi KO, Lee I, Paik SY, Kim DE, Lim JD, Kang WS, Ko S. Ultrafine Angelica gigas powder normalizes ovarian hormone levels and has antiosteoporosis properties in ovariectomized rats: particle size effect. J Med Food. 2012; 15(10):863-72.

34. Branham WS, Dial SL, Moland CL, Hass BS, Blair RM, Fang H, Shi L, Tong W, Perkins RG, Sheehan DM. Phytoestrogens and mycoestrogens bind to the rat uterine estrogen receptor. J Nutr. 2002;132(4):658-64.

35. Chang BY, Kim DS, Kim HS, Kim SY. Evaluation of estrogenic potential by herbal formula, HPC 03 for in vitro and in vivo. Reprod. 2018;155(2):105-15.

36. Jiang C, Guo J, Wang Z, Xiao B, Lee HJ, Lee EO, Kim SH, Lu J. Decursin and decursinol angelate inhibit estrogen-stimulated and estrogen-independent growth and sunvival of breast cancer cells. Breast Cancer Res: BCR. 2007;9(6):R77.

37. Giudice LC. Potential biochemical markers of uterine receptivity. Hum Reprod. 1999;14(Suppl 2):3-16.

38. Achache $\mathrm{H}$, Revel A. Endometrial receptivity markers, the journey to successful embryo implantation. Hum Reprod Update. 2006;12(6):731-46.

39. Choi HJ, Chung TW, Park MJ, Kim HS, You S, Lee MS, Joo BS, Lee KS, Kim KJ, Wee $\mathrm{G}$, et al. Benzoic acid enhances embryo implantation through LIFdependent expression of integrin alphaVbeta3 and alphaVbeta5. J Microbiol Biotechnol. 2017;27(4):668-77.

40. Whirledge SD, Oakley RH, Myers PH, Lydon JP, DeMayo F, Cidlowski JA. Uterine glucocorticoid receptors are critical for fertility in mice through control of embryo implantation and decidualization. Proc Natl Acad Sci U S A. 2015;112(49):15166-71.

41. Polak de Fried E, Blanco L, Lancuba S, Asch RH, Improvement of clinical pregnancy rate and implantation rate of in-vitro fertilization-embryo transfer patients by using methylprednisone. Hum Reprod. 1993;8(3):393-5 .

42. Ma Y, Jung JY, Jung YJ, Choi JH, Jeong WS, Song YS, Kang JS, Kaishun B, Kim MJ. Anti-inflammatory activities of coumarins isolated from Angelica gigas Nakai on LPS-stimulated RAW 264.7 cells. Prev Nutr Food Sci. 2009;14(3):179-87.

43. Ruhaak LR, Uh HW, Deelder AM, Dolhain RE, Wuhrer M. Total plasma Nglycome changes during pregnancy. J Proteome Res. 2014;13(3):1657-68.

44. Zhu ZM, Kojima N, Stroud MR, Hakomori S, Fenderson BA. Monoclonal antibody directed to Le(y) oligosaccharide inhibits implantation in the mouse. Biol Reprod. 1995;52(4):903-12.

45. Sohel MM, Hoelker M, Noferesti SS, Salilew-Wondim D, Tholen E, Looft C, Rings F, Uddin MJ, Spencer TE, Schellander K, et al. Exosomal and nonExosomal transport of extra-cellular microRNAs in follicular fluid: implications for bovine oocyte developmental competence. PLoS One. 2013;8(11):e78505.
46. Santonocito M, Vento M, Guglielmino MR, Battaglia R, Wahlgren J, Ragusa M, Barbagallo D, Borzi P, Rizzari S, Maugeri M, et al. Molecular characterization of exosomes and their microRNA cargo in human follicular fluid: bioinformatic analysis reveals that exosomal microRNAs control pathways involved in follicular maturation. Fertil Steril. 2014;102(6):1751-61 e1751.

47. da Silveira JC, Veeramachaneni DN, Winger QA, Carnevale EM, Bouma GJ. Cell-secreted vesicles in equine ovarian follicular fluid contain miRNAs and proteins: a possible new form of cell communication within the ovarian follicle. Biol Reprod. 2012;86(3):71.

48. Bidarimath M, Khalaj K, Kridli RT, Kan FW, Koti M, Tayade C. Extracellular vesicle mediated intercellular communication at the porcine maternal-fetal interface: a new paradigm for conceptus-endometrial cross-talk. Sci Rep. 2017;7:40476.

49. An X, Liu X, Zhang L, Liu J, Zhao X, Chen K, Ma H, Li G, Cao B, Song Y. MiR449a regulates caprine endometrial stromal cell apoptosis and endometrial receptivity. Sci Rep. 2017;7(1):12248.

\section{Publisher's Note}

Springer Nature remains neutral with regard to jurisdictional claims in published maps and institutional affiliations.

\section{Ready to submit your research? Choose BMC and benefit from:}

- fast, convenient online submission

- thorough peer review by experienced researchers in your field

- rapid publication on acceptance

- support for research data, including large and complex data types

- gold Open Access which fosters wider collaboration and increased citations

- maximum visibility for your research: over $100 \mathrm{M}$ website views per year

At BMC, research is always in progress.

Learn more biomedcentral.com/submissions 